\title{
(Un)gefragte Expert_innen: kindliche Erfahrungen im nachhaltigen Urbanismus
}

Rezension zu Pia Christensen / Sophie Hadfield-Hill / John Horton / Peter Kraftl

(2018): Children living in sustainable built environments. New urbanism, new citizens. Abingdon/New York: Routledge.

\section{Antonia Appel}

Abb. 1 Titel des Buches (Quelle: Routledge)

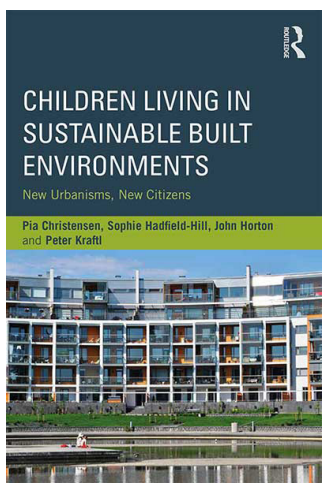

Immer mehr Städte und Gemeinden werden im Zeichen der Nachhaltigkeit geplant oder umgestaltet. Nachhaltiger Urbanismus ist mittlerweile längst kein Nischenthema mehr für klassisch „grüne“ Städte wie Freiburg oder Kopenhagen, sondern er ist zum Mainstream geworden. Die Merkmale dieses neuen urbanen Leitbildes sind besonders auf stadtplanerischer Ebene ergiebig erforscht worden (vgl. beispielsweise Farr 2012; Flint/Raco 2012; Wheeler/Beatley 2014; Rapoport 2015). Wie sich das städtische Alltagsleben in tatsächlich existierenden nachhaltig gebauten Orten darstellt, bleibt jedoch oft unbeleuchtet. Insbesondere trifft dies auf die lebensweltlichen Realitäten der jüngsten Stadtbewohner_innen zu, deren Perspektive meistens unbeachtet bleibt: Kinder scheinen im Kontext nachhaltiger Städte und Stadtentwicklung mittendrin und trotzdem nur dabei zu sein.

Im Zeichen dieses Spannungsfeldes nimmt der Band Children living in sustainable built environments die Lesenden mit auf eine Reise in vier Modellkommunen im Südosten Englands, die gänzlich im Sinne der Nachhaltigkeit geplant und gebaut wurden: Photovoltaikanlagen, Windturbinen oder nachhaltige Entwässerungssysteme sind allgegenwärtig, Gemeindezentren präsentieren sich als soziale Mittelpunkte für alle Bewohnenden und das Straßennetz ermöglicht eine möglichst große walkability. Doch die Betrachtung der nachhaltigen Architektur in den jeweiligen Gemeinden stellt nur den Beginn der Reise dar. Über fünf Kapitel tauchen wir tief in die Lebenswelten und alltäglichen Praktiken der jüngsten Bewohner_innen dieser Gemeinden ein. Verwendet werden hierfür Daten des vierjährigen Projekts New urbanism, new citizens (2009-2013) des interdisziplinären Kindheitsforscher_innenteams der Soziologin Pia Christensen und den Geograf_innen Sophie Hadfield-Hill, John Horton und Peter Kraftl. Für anschauliche Beispiele sorgen Interviewmaterial, Feldnotizen und Fotos der Autor_innen, die teilweise ein halbes Jahr in den Gemeinden verbrachten. Lesende bekommen so vielfältige Einblicke, wie es ist, als Kind an einem solchen Ort aufzuwachsen, zu leben und regelmäßig übersehen zu werden.

Ziel des Buches ist es, eine kritische Auseinandersetzung mit Überschneidungspunkten von nachhaltigem Urbanismus und Kindheit zu 
ermöglichen. Ganz im Zeichen der „new wave” der Childhood Studies (Christensen et al. 2018: 22), in der die Autor_innen ihre Forschung verorten, spielen neben alltäglichen Erfahrungen der Kinder auch verschiedenste Materialitäten und nicht-menschliche Akteur_innen - angefangen beim Straßenbelag über die Wärmepumpe bis zur Hauskatze - eine zentrale Rolle, um die Lebenswelt der jüngsten Bewohner_innen zu erfassen. So kann das Buch als empirischer Vorstoß in dieser neuen Welle interdisziplinärer Kindheitsforschung gewertet werden. Komplementär hierzu entstand in den letzten Jahren - sowohl von den Autor_innen des Buches selbst als auch von anderen Kindheitsforscher_innen - eine Reihe theoretisch geprägter Arbeiten, bestimmt von Impulsen des Posthumanismus und des neuen Materialismus (Malone/Tesar/Arndt 2020; Kraftl 2020; Spyrou/Rosen/ Cook 2019).

Die einführenden Kapitel des Buches ermöglichen einen behutsamen, jedoch lehrreichen Einstieg in diese neuen Theorieanstöße für die Kindheitsforschung wie auch in die Geschichte des nachhaltigen Urbanismus. Um Überschneidungspunkte dieser beiden Hauptkomponenten des Buches herauszuarbeiten, führen die Autor_innen ihre Lesenden souverän durch ein Dickicht von Konzepten wie Mobilitäten, Intersektionalität/Intergenerationalität, Emotionen/Affekt, Embodiment und Materialitäten. Diese fünf Konzepte bilden den Rahmen für die im Buch immer präsente Suche nach vitality, also nach Elementen, die das Dasein von Kindern in nachhaltigen Gemeinden mit Leben füllen. Dies ist bemerkenswert, setzen sich Arbeiten rund um Nachhaltigkeit und Kinder doch meist mit dem Nachhaltigkeitslernen in formalen oder non-formalen Lernkontexten auseinander (vgl. Somerville/Green 2015), nicht mit dem Einfluss alltäglicher Begegnungen.

Im Laufe des Buches stellen die Autor_innen durch detaillierte Beschreibungen deutlich heraus, dass Kinder keineswegs nur passive, individuelle Empfänger_innen sind, deren Praktiken durch nachhaltig gestaltete Räume bestimmt werden. Sie zeigen vielmehr, dass Kinder in einer aktiven Rolle als Raumaneigner_innen, Pionier_innen und Eisbrecher_innen - verbandelt in einem Netz mit ebenjenen Materialitäten und nicht-menschlichen Akteuren - ihre ganz eigene Form des nachhaltigen Urbanismus produzieren. Der Weg dahin ist jedoch geprägt von Ambivalenzen, Bevormundung und einer weitgehenden Marginalisierung.

Aus einer der vier Gemeinden berichten die Autor_innen beispielsweise von shared surfaces, die - weg von der Dominanz des motorisierten Individualverkehrs - öffentliche Wege und Straßen demokratisieren sollen. Im Sinne dieser Demokratisierung wurden Räume uneindeutig gestaltet und stehen somit zur Aushandlung zwischen den verschiedenen Nutzer_ innengruppen bereit. Es wurden keine Verkehrsschilder installiert, sondern mögliche Übergänge von Gehweg und Straße lediglich durch unterschiedliche Bodenmaterialien gekennzeichnet. Doch genau diese unklare Verkehrsführung verunsicherte Kinder in dieser Modellkommune: Wo (über-)sieht man sie? Was ist nun genau Straße? Wann halten Autos an? Diese Unklarheiten führten teilweise zu gefährlichen Situationen bis hin zu Unfällen (Christensen et al. 2018: 99). Was auf planerischer Ebene als größere Bewegungsfreiheit und nachhaltige Teilhabe von Kindern angedacht war - deren Mobilität vom $\mathrm{Zu}-\mathrm{Fu} \beta-G e h e n$ und Fahrradfahren geprägt ist -, befeuerte in der 
Realität genau das Gegenteil. Dieses komplexe Zusammenwirken aus dem Straßenbelag, fehlenden Schildern, dem gemeinsamen (Un-)Verständnis der Straße, verschiedenen Verkehrsteilnehmer_innen und den körperlichen Erfahrungen in potenziell gefährlichen Situationen steht sinnbildlich für die vielschichtigen Beziehungen, die Kinder tagtäglich in nachhaltigen Umgebungen eingehen (müssen).

Das Außer-Acht-Lassen von Kindern zieht sich als alltägliche Erfahrung, auch in Form sozialer und räumlicher Ausgrenzung, wie ein roter Faden durch das Buch. Es fängt an bei einem Gemeindezentrum, beworben als Mittelpunkt der Gemeinde, in dem fünf von insgesamt sieben Regeln an Kinder gerichtet sind, ihnen etwa nur in Begleitung eines Erwachsenen Zutritt gewährt wird, und hört auf bei Planungsprozessen für die Weiterentwicklung der Gemeinden, bei denen Perspektiven von Kindern gänzlich fehlen und/oder Kinder lediglich durch Erwachsene vertreten werden (ebd.: 134). Ernüchtert stellen die Autor_innen fest, dass Kinder zwar die Werbegesichter nachhaltiger Kommunen seien, sich hinter dieser beworbenen Familienfreundlichkeit jedoch vor allem eine Erwachsenenzentrierung verberge.

Besonders frustrierend sind diese Feststellungen, wenn man die Berichte der Kinder liest, die laufend ihre Umwelt erkunden, ungeahnte Wege gehen und Grenzen überschreiten - wodurch sie zu denjenigen Bewohner_innen werden, die am meisten lokales Expert_innenwissen ansammeln und so als unsichtbare community builders agieren.

„We have shown how children have a central, constitutive role in welcoming neighbours and opening up urban spaces to community use, engagement and senses of vitality. Children and young people's everyday narratives, knowledges and mobilities were (and are) key to the development of such senses of community in new urban spaces [...]. Underpinning these data was also a sense of the considerable, detailed knowledges that children and young people frequently produce about new urban spaces. However, as we have already argued, adult planners and decision-makers have rarely engaged with this kind of expertise and specialist knowledge.“ (Ebd.: 128)

In Anbetracht dieser Erkenntnisse rufen die Autor_innen ganz praktisch und konkret dazu auf, Kindern einen Platz am Tisch von Entscheidungsprozessen einzuräumen, und präsentieren hierfür einen Leitfaden (ebd.: 180 ff.). Zudem plädieren sie für weitere Forschung im Kontext neuer urbaner Nachhaltigkeit. Hierfür werfen sie eine Reihe an Forschungsfragen auf, die sich im Rahmen der neuen Welle der Kindheitsforschung stellen. Neben Anknüpfungspunkten zu nachhaltiger Mobilität von Kindern oder zum Austarieren von Stimmrechten menschlicher und nicht-menschlicher Akteur_innen sind hier auch Fragen übergeordneter Natur präsent: Können Theorie und Praxis wirklich im Sinne der neuen Welle der Kindheitsforschung zusammengeführt werden? Wie kann dies geschehen? So spannend die neuen Impulse in der Kindheitsforschung seien, so vorsichtig müsse gleichzeitig ausgelotet werden, wie und wo diese theoretischen Ansätze in der Praxis überhaupt fruchtbar gemacht werden können.

Diskussionen rund um den viel umstrittenen Begriff der Nachhaltigkeit meiden die Autor_innen: Sie geben an, nur Orte, Architekturen und Policies 
berücksichtigt zu haben, die in offiziellen Dokumenten als nachhaltig bezeichnet werden, und merken an, dass dies durchaus streitbar sei. Zwar finden sich vereinzelt Kritikpunkte, beispielsweise an den häufigen Verflechtungen von Nachhaltigkeit und neoliberalen Praktiken oder am engen Nachhaltigkeitsverständnis von Bildung für nachhaltige Entwicklung. Eine grundsätzlichere kritische Auseinandersetzung mit dem Begriff bleiben die Autor_innen jedoch schuldig. Allerdings sorgt diese Vermeidung auch dafür, dass sich das Buch nicht im Minenfeld der Nachhaltigkeitsdiskussion verliert und sich an der Definition des Begriffes aufhängt, wodurch das Hauptaugenmerk auf die Erfahrungen und Lebenswelten junger Menschen gerichtet werden kann.

Die Besonderheit und große Stärke des Buches sind ohneZweifel die vielen anschaulichen und vor allem alltäglichen Beispiele aus den Gemeinden. Sie führen vor Augen, dass es dringend notwendig ist, der Tatsache Rechnung zu tragen, dass sich Alltage, Wirklichkeiten und Praktiken von Kindern durch einen weltweiten Wandel hin zur Nachhaltigkeit im Urbanen verändern. Die Ergebnisse werden in eine internationale und vor allem nicht-westliche Perspektive gesetzt, indem immer wieder Berichte einer ähnlichen Studie zu Kindern in nachhaltigem, neuem Urbanismus in Indien eingestreut werden (Hadfield-Hill/Zara 2017). Dadurch wird deutlich, dass nachhaltiger Urbanismus zwar ein weltweites Phänomen ist, jedoch lokal verschiedenste Ausprägungen hat und somit unterschiedlich auf den Alltag von Kindern wirkt. Gerade weil das Buch die globale Natur des urbanen Wandels hin zur Nachhaltigkeit und die damit verbundenen Konsequenzen für die jüngsten Stadtbewohner_innen weltweit betont, hätte ein etwas ausführlicheres InPerspektive-Setzen diesen Aspekt noch mehr hervorheben können.

Um kindliche Praktiken im Kontext von nachhaltigem Urbanismus greifbarer zu machen, bieten die Autor_innen zum Schluss eine inspirierende Perspektive an: Spiel. Fokussiert werden hierbei insbesondere die Räume zwischen Kindheit und Spiel: Spielend werden Kontakte geknüpft, neue Räume erkundet, Grenzen ausgetestet oder überschritten und Beziehungen mit nicht-menschlichen Dingen aufgebaut.

„[...] the space between childhood and play is a cavernous, complex and contested one. Importantly, it is one in which many of the concerns of city life - of equitable, just, and essentially sustainable city lives - are played out. Children and/or play may recede into the background, but understood in the non-dualistic, non-essentialised, non-romanticised ways we argued for [...], they nevertheless provide key lynchpins for articulating richer and more diverse senses of what matters in the production of sustainable vitalities." (Christensen et al. 2018: 171; Herv. i. O.)

Hiermit wird der Bogen zurück zum Beginn des Buches geschlagen, als sich die Autor_innen auf die Suche nach der vitality von nachhaltigem Urbanismus machten. Das Aufsetzen einer Spiel-Brille ermöglicht hierbei eine klarere Sicht und stößt die Tür für weitere Forschung über Kinder in nachhaltig gebauten Umgebungen noch ein Stück weiter auf. Das Buch stellt einen gelungenen Ausgangspunkt für dieses komplexe Unterfangen bereit. Die gewonnenen Erkenntnisse über lebensweltliche Realitäten von Kindern 
in nachhaltigen Umgebungen regen an, diese im Zeichen der new wave der Kindheitsforschung zu vertiefen und bieten vielerlei Anknüpfungspunkte für Forschungen zu Kindern in der nachhaltigen Stadt. Am Ende erscheint es nur sinnvoll, einen nachhaltigen und damit zukunftsfähigen Lebensstil in nachhaltig gebauten Umgebungen auch durch die Augen der zukünftigen Generation zu betrachten.

Die Publikation wurde mit Mitteln der Deutschen Forschungsgemeinschaft (SCHR 1329/1-1) gefördert.

\section{Autor_innen}

Antonia Appel ist Geographin. Sie forscht im Bereich der geographischen Kindheitsforschung an der Schnittstelle von Bildungs- und Stadtgeographie im Kontext von Nachhaltigkeit. antonia.appel@ph-freiburg.de

\section{Literatur}

Christensen, Pia / Hadfield-Hill, Sophie / Horton, John / Kraftl, Peter (2018): Children living in sustainable built environments. New urbanisms, new citizens. Abingdon/New York: Routledge.

Farr, Douglas (2012): Sustainable urbanism. Urban design with nature. Hoboken: John Wiley \& Sons.

Flint, John / Raco, Mike (Hg.) (2012): The future of sustainable cities. Critical reflections. Bristol: Policy Press.

Hadfield-Hill, Sophie / Zara, Cristiana (2017): Final report. New urbanisms in India. Urban living, sustainability, everyday life. Birmingham: University of Birmingham.

Kraftl, Peter (2020): After childhood. Re-thinking environment, materiality and media in children's lives. London: Routledge.

Malone, Karen / Tesar, Marek / Arndt, Sonja (2020): Theorising posthuman childhood studies. Singapore: Springer Nature.

Rapoport, Elizabeth (2015): Globalising sustainable urbanism. The role of international masterplanners. In: Area 47/2, 110-115.

Somerville, Margaret / Green, Monica (2015): Children, place and sustainability. London: Palgrave Macmillan.

Spyrou, Spyros / Rosen, Rachel / Cook, Daniel Thomas (Hg.) (2019): Reimagining childhood studies. London: Bloomsbury Academic.

Wheeler, Stephen M. / Beatley, Timothy (2014): Sustainable urban development reader. Hoboken: Taylor and Francis. 